\title{
Chemically Imaging the Interaction of Acetylated Nanocrystalline Cellulose (NCC) with a Polylactic Acid (PLA) Polymer Matrix
}

Tapasi Mukherjee ${ }^{* 1}$, Mark J. Tobin ${ }^{2}$, Ljiljana Puskar ${ }^{3}$, Marc-Antoine Sani ${ }^{4}$, Nhol Kao ${ }^{1}$, Rahul K. Gupta', Muthu Pannirselvam ', Nurul Quazi ${ }^{1}$ and Sati Bhattacharyal

1. Rheology and Materials Processing Centre, School of Civil, Environmental and Chemical Engineering, RMIT University, Melbourne, Victoria 3001, Australia.

2. Australian Synchrotron, 800 Blackburn Road, Clayton, Victoria 3168, Australia.

3. Methods for Material Development, Helmholtz-Zentrum für Materialien und Energie GmbH, Berlin, 12489 Germany,

4. Bio21 Molecular Science and Biotechnology Institute, The University of Melbourne, Parkville, Melbourne, Victoria 3010, Australia.

E-Mails: tapashi.mukherjee@rmit.edu.au; mark.tobin@synchrotron.org.au;

ljiljana.puskar@helmholtz-berlin.de; msani@unimelb.edu.au; nhol.kao@rmit.edu.au; rahul.gupta@rmit.edu.au; muthu.pannirselvam@rmit.edu.au; nurul.quazi@rmit.edu.au; sati.bhattacharya@rmit.edu.au

KEYWORDS: FTIR, NMR, Chemical Imaging, Dispersion, Nanocrystalline Cellulose (NCC), Poly (lactic acid) 


\begin{abstract}
The non-covalent interaction of acetylated nanocrystalline cellulose (AC-NCC) with polylactic acid (PLA) in a composite blend has been studied at the micron scale by synchrotron Fourier Transform Infrared (FTIR) microspectroscopy. Microtomed sections of AC-NCC in PLA showed strong, localized carbonyl stretching $(\mathrm{v}=\mathrm{O})$ absorbance characteristic of the cellulose acetylation, and this was observed on the surface of larger aggregated AC-NCC particles. A shift in the $v \mathrm{C}=\mathrm{O}$ IR absorption peak of $\mathrm{AC}-\mathrm{NCC}$ in PLA, relative to unblended AC-NCC was observed, which is indicative of an intermolecular interaction between AC-NCC and PLA matrix. Acetylation can therefore potentially improve the performance of the composite by enabling linkages between carbonyl groups, helping to establish a good stress transfer between the fiber and the matrix. This could in turn lead to a material with high yield elastic modulus. This is the first reported chemical imaging of acetylated nanocrystalline cellulose-based composite materials using synchrotron FTIR microspectroscopy.
\end{abstract}

\title{
1. Introduction
}

In polymer composites, the interface between the composite components plays a defining role in the overall material properties such as: glass transition temperature (Rittingstein et al. 2007 and Roth et al. 2007) relaxation dynamics (Labardi et al. 2010 and Priestley et al. 2005), aging (Rittingstein and Torkelson. 2006), dielectric behaviour such as breakdown strength, voltage endurance, and dielectric permittivity (Roy et al. 2005), density (Ciprari et al. 2006), and mechanical properties such as stiffness, debonding, fracture, internal stress distribution and toughness (Needleman et al. 2010 and Ciprari et al. 2006), and flammability(Kashiwagi et al. 2005). Theoretical and experimental studies in this area suggest that the structure and properties 
of the matrix transition layer (interface) play the main role in the noncovalent interaction between nanoparticles and the polymer matrix (Priestley et al. 2005). A strong interaction of two phases (polymeric substrate and polymeric nanoparticle) results in the mobility of matrix molecules and their conformations being observed in the transition layer. The interfacial interactions play a very important role for processing composites. This is applicable for wetting the filler or fiber with the molten polymer (thermoplastic based composites) or reactive systems (thermosets and some thermoplastic-based composites), as well as the long term physical properties described earlier (Ching et al. 2016). The interfacial zones and surface characteristics play a predominant role for designing specific morphologies like the dispersion state in such composite system. Overwhelmingly, research in this area suggests that filler size, loading and distribution dictate the level of interaction between the filler and matrix, while the surface structure and chemistry of the particles dictate the intensity of that interaction at the particle/polymer interface (Khare and Burris. 2010). The successful application of nanocomposite technology in novel composite materials requires the development of a more fundamental understanding of the reinforcement mechanisms.

Polylactic acid or polylactide (PLA) based bio-composites offer hope for designing and developing sustainable biomedical implants like artificial joints and tissues (Evans et al. 2000). An important aspect in designing such implants lies in their biocompatibility, together with their strength and physical properties, as compared to natural ligaments and tendons. Nanocrystalline Cellulose (NCC) is a powerful reinforcing agent, primarily because of its high strength and elasticity (Huda et al. 2006). Although NCC offers a potential reinforcement to the PLA matrix, the challenge lies in dispersing the particles sufficiently well in the polymer matrix. The hydroxyl groups, as present on the surface of NCC, limit its dispersion by agglomeration, leading 
to cracking or failure of the composites (Eichorn et al. 2010). Improving the dispersion of the cellulose fibres in the biopolymer matrix is therefore an active area of research interest. A possible way to achieve a good dispersion during processing is to block cellulose fibre selfinteractions by binding them with a hydrogen bond-forming solvent. Several "solubilising schemes" have been explored over the last decade to improve dispersion which include the use of surfactants (Oksman et al. 2006), acetylation (Braun and Dorgan. 2009), silylation (Pei et al. 2010), and grafting (Pracella et al. 2010).

FTIR microspectroscopy is a well-established technique for the chemical identification of materials and for helping to visualise the distribution of components within complex compounds such as biological tissues or composite materials (Heraud et al. 2010 and Russell et al. 2014). Typical applications of FTIR microspctroscopy include the examination of the homogeneity of coatings and the analysis of the distribution of a multitude of different components in complex mixtures (Clemons et al. 2013 and Duncan and Williams. 1983). The molecules or functional groups are identified on the basis of characteristic vibrations of bonds or groups of bonds when excited by the impinging IR beam. In this way, FTIR microspectroscopy delivers information about the molecular composition of the analysed sample area. Spatially resolved data sets of multiple IR spectra can be collected, with a spatial resolution between sampling positions of a few microns, and maps of the distribution of chemical groups identified within the IR spectra can be generated from these data sets. For this reason, this technique is also referred to as "chemical imaging". A significant improvement in the quality of data obtained from the smallest of samples can be achieved by the use of synchrotron radiation as the infrared source for FTIR microspectroscopy. A synchrotron infrared source is typically 100 times brighter than a 
conventional thermal (e.g. Globar) source and the high brightness (i.e. flux density) of the source results in all of the available infrared light being focused into a diffraction-limited spot on the sample of 5 to 10 microns in size. This allows smaller regions to be probed with high signal-tonoise ratio. The Australian Synchrotron Infrared Microspectroscopy (IRM) beamline allows the acquisition of high signal-to-noise mid-infrared absorbance data from diffraction-limited spots within the sample, and has been used to study a wide range of materials, from live biological cells to paintings (Tobin et al. 2010 and Osmond et al. 2012).

In univariate mode, FTIR spectral analysis provides information on functional groups based on absorption band intensities, band areas and band positions, or the ratio of band intensities or areas. Images can then be generated by plotting the unique frequencies as a function of spatial position and spectral intensity. Second derivative analysis of each spectral data set may be useful to reveal the spectral position and spatial distribution of weaker or overlapping absorption peaks enabling subtle spectral differences within the spectral data set to be further analysed (Wang et al. 2010).

In the study reported here, attempts have been made to partially substitute by esterification the hydroxyl groups present on the surface of nanocrystalline cellulose. A series of nanocomposites has been subsequently prepared by solvent casting acetylated NCC (1-10 wt. \%) in the PLA matrix using dichloromethane as the solvent. It was previously reported that increase in the density of ester linkages from the polymer matrix to the cellulose interface contributes to an increase in the elastic modulus and adhesion property within the resulting material (Pakzad et al. 2012). A characteristic IR absorbance peak of esterification is the $v C=O$ stretch occurring in the 
range between $1800 \mathrm{~cm}^{-1}$ and $1650 \mathrm{~cm}^{-1}$ such as that observed in the study of coupling mechanisms and interfacial characterization on maleated wood-fiber/high density polyethylene composites ( $\mathrm{Lu}$ et al. 2005). Here we describe the use of synchrotron FTIR microspectroscopy to map the spatial distribution of the functional group chemistry of the composite constituents. The composite chosen for this purpose was one in which the particulate acetylated NCC is more or less uniformly dispersed in the PLA matrix, but in which some remaining aggregation resulted in particles of a few microns in size, which could be observed by optical microscopy and analysed by FTIR microspectroscopy. The main objective of the study was to understand the role played by carbonyl groups in the interfacial interaction between the fibre and the matrix, thereby improving the overall adhesion and mechanical properties of the composites. Second derivative analysis of the carbonyl absorption peak across sections of the composite material, at the micron scale was performed to investigate possible chemical interaction of the carbonyl group at the interfacial region of the matrix.

\section{Materials and Methods}

\subsection{Materials}

A polylactic acid biopolymer (Nature Work PLA Polymer 4032D) with a molecular weight about 180,000 , a density of $1.24 \mathrm{~g} \mathrm{~cm}^{-3}$ and a melting point of $160{ }^{\circ} \mathrm{C}$ was chosen as the matrix. Microcrystalline cellulose was purchased from Sigma Aldrich (Castle Hill, NSW, Australia) and nanocrystalline cellulose was prepared by acid hydrolysis of the microcrystalline cellulose. Acetic anhydride, sodium hydride, and dimethyl formamide and dichloromethane (DCM) were purchased from Sigma Aldrich. 


\subsection{Sample Preparation}

\subsubsection{Acetylation of NCC.}

In a one litre round bottom flask, $15 \mathrm{~g}$ of freeze dried NCC and $100 \mathrm{ml}$ of dimethyl formamide were stirred at $60{ }^{\circ} \mathrm{C}$ for half an hour. Sodium Hydride (7 g, $60 \%$ dispersion in mineral oil) was added portion wise in the reaction mixture and stirring was continued overnight $(16 \mathrm{~h})$ in a nitrogen atmosphere. Next day, acetic anhydride $(10 \mathrm{ml})$ was added and stirred for further 3 hours.

The above reaction mixture was cooled to room temperature with continuous stirring. Finally ethanol $(50 \mathrm{ml})$ was added carefully to deactivate excess sodium hydride. The reaction mixture was poured into $400 \mathrm{ml}$ of water and stirred; the solid was collected by filtration and washed with water and acetone, dried at $80{ }^{\circ} \mathrm{C}$ in a vacuum oven, to give the acetylated $\mathrm{NCC}(\sim 15 \mathrm{~g})$. The surface acetylated NCC, referred to as AC-NCC, was oven dried in vacuum at $70{ }^{\circ} \mathrm{C}$ for 24 hours.

\subsubsection{Preparation of Composites.}

The desired amount of AC-NCC was added along with $20 \mathrm{~g}$ of PLA into DCM solvent (200$400 \mathrm{ml}$ approx.) with mechanical stirring to produce a mixture, which formed a viscous solution over a period of $5 \mathrm{hrs}$. The mixture was conditioned overnight to eliminate bubbles and was then cast into a Petri dish. DCM was allowed to evaporate off at ambient temperature (ca. $25^{\circ} \mathrm{C}$ ) for approx. 24 hours. Finally, the solidified films, with a thickness of about $0.5 \mathrm{~mm}$, were vacuum dried overnight, and then kept in a desiccator containing silica gel. The resultant composite sheets were coded as PLA-AC-NCC (10 wt. \%). Cross-sections approximately $2.5 \mu \mathrm{m}$ in thickness were cut with a microtome from the films and used for the FTIR analysis. This 
thickness was selected to ensure that the known strong $v \mathrm{C}=\mathrm{O}$ IR absorption observed in these samples produced an absorbance value of less than $1.0 .10 \mathrm{wt} . \%$ was found to be a concentration which ensured the presence of adequate numbers of observable micron-scale particles in the individual microtomed sections that were examined, and all results reported here are therefore for samples of 10 wt. \% AC-NCC in PLA.

\subsection{Characterization}

\subsubsection{Fourier Transform Infrared Microspectroscopy.}

Pure samples of NCC and AC-NCC were analysed by FTIR microspectroscopy after pressing small quantities of each material between the windows of a diamond Micro Compression cell (Thermo Fisher Scientific Inc., Waltham, MA), with a small KBr crystal mounted next to the sample as reference, ensuring good contact between the sample section and the diamond windows. Individual microtomed PLA composite sections were mounted in the Micro Compression cell for analysis, in the same manner as used for the pure component samples. The $\mathrm{KBr}$ reference crystal was used for collection of background spectra in all cases rather than collection through air adjacent to the sample so as to reduce spectral artefacts resulting from optical interference that can arise from back reflections within the air gap between the two diamonds.

Prior to FTIR microanalysis, sections were observed by bright field visible light microscopy using crossed polarized illumination, allowing individual crystallites of NCC or AC-NCC to be identified within the section, due to their birefringence. In the case of AC-NCC, the presence of clearly observable crystalline cellulose particles of between $1 \mu \mathrm{m}$ and $10 \mu \mathrm{m}$ in size within the 
composite shows that some degree of aggregation of the AC-NCC has occurred during preparation, and is an indicator that the acetylation of the cellulose hydroxyl groups was only partially complete. Unacetylated cellulose particles were found to be larger in size than acetylated cellulose, indicating a higher level of aggregation for NCC. Particles identified by cross polarized illumination were selected for FTIR microanalysis.

High spatial resolution IR maps were recorded at the IRM beamline at the Australian Synchrotron. The beamline operates a Bruker V80v vacuum FTIR spectrometer, linked to a Bruker Hyperion 2000 IR microscope (Bruker Optics, Ettlingen, Germany). The microscope is equipped with a $36 \times(0.5$ numerical aperture $)$ objective and condenser and a narrow band mercury cadmium telluride detector, sensitive in the mid-IR spectral region to $750 \mathrm{~cm}^{-1}$. The infrared beam is focused onto the sample with a spot size of approximately $8 \times 8$ microns, and the area of analysis can be further refined, using apertures, to about $3 \times 3$ microns. A motorized stage allows spectral mapping by raster scanning the sample through the focused beam. For the work reported here the aperture size for analysis was $5 \times 5$ microns and the mapping step size was $2 \times 2$ microns. FTIR maps were collected such that they covered an area including one or more particles of NCC or AC-NCC. Spectra were co-added for 16 scans at each sample position and 64 scans in the background position with a spectral resolution of $4 \mathrm{~cm}^{-1}$. Data collected at the beamline were analyzed using the Bruker software OPUS 7.2.

Spatial distribution of the aliphatic C-H stretching absorbance of the $\mathrm{CH}_{2}$ functional group $\left(\mathrm{vCH}_{2}\right)$, which is present in NCC and AC-NCC but absent from PLA, was used to confirm the location and identity of the selected birefringent particles as AC-NCC or NCC by plotting a map of the integrated spectral peak area between $2938 \mathrm{~cm}^{-1}$ and $2860 \mathrm{~cm}^{-1}$ for each $2 \mathrm{D}$ data set. 
Analysis of the peak position of the $\mathrm{v}=\mathrm{O}$ carbonyl peak of FTIR spectra from particles and the surrounding PLA matrix was achieved by second derivative analysis, applying a 9 point Savitsky-Golay smoothing function, using the Bruker OPUS 7.2 software. Second derivative spectra contain negative peaks corresponding to peaks and shoulders in the original absorbance spectra. However, to enable $2 \mathrm{D}$ spectral maps of the integrated negative second derivative $v \mathrm{C}=\mathrm{O}$ peaks to be displayed using the same colour scale as maps of the integrated positive $v \mathrm{CH}_{2}$ absorbance peaks, all second derivative spectra were inverted by multiplying by -1 to produce positive peaks. All second derivative spectra are therefore displayed as inverted spectra too.

\subsection{2 ${ }^{13}$ C Solid State NMR.}

Solid-state cross-polarization magic angle spinning carbon-13 nuclear magnetic resonance (CP/MAS $\left.{ }^{13} \mathrm{C}-\mathrm{NMR}\right)$ spectroscopy was performed on a VNMRS $600 \mathrm{MHz}$ NMR spectrometer equipped with $4 \mathrm{~mm}$ CPMAS triple resonance probe. The ${ }^{13} \mathrm{C}$ frequency was $150.8 \mathrm{MHz}$ and the chemical shift resonances were referenced to the adamantane chemical shifts obtained with a similar pulse sequence. Acquisition was performed with a CP pulse sequence using a $5.25 \mathrm{~ms}$ proton $\pi / 2$ excitation pulse, a spectral width of $50 \mathrm{kHz}$, an acquisition time of $25 \mathrm{~ms}$ using a SPINAL-64 decoupling scheme of $70 \mathrm{kHz}$ strength and a $5 \mathrm{~s}$ delay between transients. The Hartmann-Hann match was optimized on the -1 sideband at a MAS speed of $8 \mathrm{kHz}$ with a contact time of $500 \mathrm{~ms}$ and a linearly ramped-CP field of $30 \pm 0.8 \mathrm{kHz}$. Typically, 12000-16000 transients were accumulated at $25^{\circ} \mathrm{C}$. A line broadening of $50 \mathrm{~Hz}$ and zero filling to $65 \mathrm{k}$ points were used to process the spectra.

\section{Results and Discussion}




\subsection{Acetylation of NCC.}

The reaction scheme for acetylation of NCC is shown in Fig. 1a, and the resulting acetylated NCC was characterized by FTIR microspectroscopy and solid-state CP/MAS ${ }^{13} \mathrm{C}$-NMR spectroscopy. FTIR absorption spectra of unreacted NCC and the resulting acetylated compound are shown in Fig.1b and are consistent with the assignment of cellulose absorption peaks reported elsewhere (Široký et al. 2010). Additional peaks were observed in the AC-NCC, indicated by arrows in Fig. $1 \mathrm{~b}$, notably a strong $\mathrm{vC}=\mathrm{O}$ peak around $1742 \mathrm{~cm}^{-1}$ and an aliphatic C-O-C stretch at around $1237 \mathrm{~cm}^{-1}$, plus a weaker peak, possibly corresponding to antisymmetric COO- vibrations, at $1573 \mathrm{~cm}^{-1}$, the presence of these functional groups together confirming the addition of the acetyl groups. Similarly, a decrease in the intensity of $-\mathrm{OH}$ stretching vibrations was observed due to successful acetyl group substitution (not shown). The observed result is similar to that reported in the study of acetylation of cellulose microcrystals reported earlier (Mukherjee et al. 2013). Fig. 1c represents the spectral curve by ${ }^{13} \mathrm{C}$ solid state NMR. With acetylation, there were two chemical shifts located at $172 \mathrm{ppm}$ and $20 \mathrm{ppm}$, as assigned to $-\mathrm{C}=\mathrm{O}$ and $-\mathrm{CH}_{3}$ stretching, thus again confirming the successful acetylation of NCC. The results obtained were similar to the observation made by Lin et al. (2011), where acetylation was performed on cellulose nanocrystals.

\subsection{Chemical Imaging.}

As described above, particles of AC-NCC and NCC were observed within the sample sections by bright field light microscopy and cross polarized light microscopy. Since individual NCC particles should be below the lateral resolution limit of light microscopy, this demonstrates that some aggregation of particles has occurred during production of the composites, and that the acetylation of $\mathrm{OH}$ group in the AC-NCC is therefore not complete, allowing a degree of 
aggregation in the AC-NCC. The AC-NCC particles observed were typically between $2 \mu \mathrm{m}$ and $20 \mu \mathrm{m}$ in size with a few particles up to $30 \mu \mathrm{m}$. The unacetylated NCC particles were generally larger in size, with few smaller $(2-5 \mu \mathrm{m})$ particles observed. The reduced particle size in the ACNCC composite nevertheless indicated improved dispersion following partial acetylation. Preliminary analysis of the IR absorbance spectra from selected particles, identified by the birefringence in under polarized illumination, confirmed these to be AC-NCC or NCC, based on the presence of $\mathrm{vCH}_{2}$ and $\mathrm{vOH}$ absorbance peaks, which are not observed in the surrounding PLA matrix. Fig. 2a shows two typical AC-NCC particles of 5-10 $\mu \mathrm{m}$ in size (outlined), with a correlated map of $\mathrm{vCH}_{2}$ absorbance, integrating the spectral peak area between $2938 \mathrm{~cm}^{-1}$ and $2860 \mathrm{~cm}^{-1}$, in Fig. 2b. Fig. 2c shows the mid-IR absorbance spectra, each averaged from the four PLA (black) and AC-NCC (orange) points marked in Fig. 2b. The strong $v \mathrm{OH}$ absorption marked in the AC-NCC spectrum in the Figure $2 \mathrm{C}$ main figure, and absent from the PLA spectrum, arises from residual un-acetylated $\mathrm{OH}$ groups of AC-NCC, and the smaller $\nu \mathrm{CH}_{2}$ absorbance arising from the cellulose side chain, and also absent from the PLA spectrum, is marked in the expanded Figure $2 \mathrm{C}$ inset spectrum.

Fig. 3a shows a microtomed section of $10 \mathrm{wt} . \%$ AC-NCC in PLA with brightfield illumination (main image), with a crossed polarized image of part of the sample shown as an inset. Individual aggregate particles of AC-NCC appear bright against a dark background in the crossed polarized image, owing to their birefringence, and several are indicated by white arrows in the polarized image. These particles are also indicated in the brightfield image where the compressed $\mathrm{KBr}$ reference crystal is shown by a black arrow. Particles for FTIR analysis were selected only from areas within the sample that were in good contact with the diamond windows of the compression cell, as indicated by bright, clear transmission in the surrounding PLA in the bright field image. 
Areas showing imperfect contact, as indicated by the rainbow-like pattern seen in the upper third of the section shown in Fig. 3a, typically showed distorted spectral profiles resulting from optical interference and particles from such regions were not analysed.

Further individual particles, or groups or particles, were selected for FTIR spectral analysis of the $v \mathrm{C}=\mathrm{O}$ absorbance peak by mapping of defined regions of the microtomed sections. An example region containing two particles is shown highlighted in Fig. 3b (yellow rectangle), and is shown at higher magnification in Fig. 3c with the two particles outlined. Fig. 3d shows an IR absorption map for the selected area, representing the integrated peak area of the second derivative spectra between $1739 \mathrm{~cm}^{-1}$ and $1754 \mathrm{~cm}^{-1}$, showing the distribution of the carbonyl absorption band associated with AC-NCC. Analysis of the so-called "fingerprint" region of the IR absorbance spectra, from $750 \mathrm{~cm}^{-1}$ to $1950 \mathrm{~cm}^{-1}$, of the PLA matrix and of selected AC-NCC particles showed many common features, and spectra are shown in Fig. 3e that are the average spectra from the measured positions indicated by black (PLA) and orange (AC-NCC) markers in Fig. 3d. Owing to the small size of the AC-NCC particle being analysed, it is possible that the spectrum attributed to $\mathrm{AC}-\mathrm{NCC}$ in Fig. 3e also includes a contribution from the PLA matrix lying above or below the particle within the microtomed section. The Fig. 3e inset shows an expanded view of the $\mathrm{vC}=\mathrm{O}$ carbonyl absorbance peaks of the PLA matrix (black) and AC-NCC particle (orange) showing that the $\mathrm{vC}=\mathrm{O}$ peak of the AC-NCC is situated at lower wavenumbers, and is broader with stronger shoulders than that of the PLA matrix. Second derivative analysis was subsequently used to determine the main peak and shoulder positions of these spectra, and Fig. 3f shows the second derivatives of the PLA matrix and AC-NCC spectra shown in Fig. 3e 
plus, for comparison, the second derivative of the pure AC-NCC spectrum previously shown in Fig. 1b. The second derivative spectrum of PLA matrix (Fig. 3f, black) shows a strong single peak at $1760 \mathrm{~cm}^{-1}$ (arrowed) and a weak shoulder at $1750 \mathrm{~cm}^{-1}$, unincorporated AC-NCC (blue spectrum) shows a broad peak at $1742 \mathrm{~cm}^{-1}$ (arrowed) and a weak shoulder at $1752 \mathrm{~cm}^{-1}$ whereas AC-NCC incorporated into PLA (orange spectrum), has a $v C=O$ peak at around $1758 \mathrm{~cm}^{-1}$ (not arrowed) and a strong shoulder at $1748 \mathrm{~cm}^{-1}$ (arrowed).

Comparing first the unblended filler and the matrix, the $v \mathrm{C}=\mathrm{O}$ peak of unincorporated AC$\mathrm{NCC}$ at $1742 \mathrm{~cm}^{-1}$ is broader, and at lower wavenumber than the $\mathrm{vC}=\mathrm{O}$ peak of PLA at $1760 \mathrm{~cm}^{-}$ ${ }^{1}$ and this difference in peak position between the two base materials can be attributed to interand intramolecular hydrogen bonding within the $\mathrm{AC}-\mathrm{NCC}$ between the $\mathrm{C}=\mathrm{O}$ of introduced acetyl groups and residual $\mathrm{OH}$ groups prior to blending. Such molecular interaction is known to lead to shift of this magnitude in the $v \mathrm{C}=\mathrm{O}$ peak (Koenig. 1999), and the necessary residual unacetylated $\mathrm{OH}$ groups are present in the AC-NCC, as already described.

The AC-NCC peak at $1758 \mathrm{~cm}^{-1}$ (Fig. 3f, not arrowed) likely contains a significant contribution from underlying PLA, as mentioned previously, and cannot confidently be attributed to the AC-NCC particle. However, the strong shoulder at $1748 \mathrm{~cm}^{-1}$ (arrowed) is specific to blended AC-NCC, lying between the $v \mathrm{C}=\mathrm{O}$ peak position of pure $\mathrm{AC}-\mathrm{NCC}$ and that of PLA.

Fig. 4 displays $v \mathrm{CH}_{2}$ and $v \mathrm{C}=\mathrm{O}$ IR absorption maps, with second derivative $v \mathrm{C}=\mathrm{O}$ spectra, for three further groups of AC-NCC particles and for two un-acetylated NCC particles, each embedded in a PLA matrix, and prepared for analysis as described previously. For each sample, an absorption map of the $\mathrm{vCH}_{2}$ peak is given (Fig. $4 \mathrm{~b}, \mathrm{f}, \mathrm{j}, \mathrm{n}$ and $\mathrm{r}$ ) adjacent to a bright field 
visible image of the particles, to confirm the chemical identity of each particle, as described above for the data shown in Fig. 2. The associated $v \mathrm{C}=\mathrm{O}$ distribution maps (Fig. 4c, g, k, o and s) are derived from integration of the second derivative spectrum around the peak or shoulder observed at $1748 \mathrm{~cm}^{-1}$ in AC-NCC, integrated between $1739 \mathrm{~cm}^{-1}$ and $1754 \mathrm{~cm}^{-1}$. The right hand column of Fig. $4(\mathrm{~d}, \mathrm{~h}, \mathrm{l}, \mathrm{p}, \mathrm{t})$ shows the second derivative spectra of $v \mathrm{C}=\mathrm{O}$ peaks between 1700 $\mathrm{cm}^{-1}$ and $1820 \mathrm{~cm}^{-1}$ from each marked position in the adjacent $\mathrm{vC}=\mathrm{O}$ absorption maps. For the AC-NCC particles shown in Fig. $4 \mathrm{c}$ and g, which are between $10 \mu \mathrm{m}$ and $30 \mu \mathrm{m}$ in size, the derivative spectra show a peak around $1758 \mathrm{~cm}^{-1}$ with a clear shoulder at $1748 \mathrm{~cm}^{-1}$ (Fig. $4 \mathrm{~d}$ and h, orange, arrowed), similar to that observed for the AC-NCC particle shown in Fig. 3. This "peak and shoulder" shape is not seen in the un-acetylated NCC particles (Fig. 4p and t, orange spectra) though a broader weak residual peak is present which accounts for the pink colour in the corresponding integrated images (Fig. $4 \mathrm{o}$ and s). The "peak and shoulder" derivative $v \mathrm{C}=\mathrm{O}$ peak shape is also seen in the third AC-NCC sample in Fig. 41, though in this case the shoulder is weaker and this may reflect the small size of the selected particles, which are $10 \mu \mathrm{m}$ or less in diameter.

The combined observation from these samples is that the PLA-incorportated AC-NCC particles show a carbonyl peak shape that is higher in wavenumber than and different in shape to that of the un-incorportated AC-NCC, resulting in a characteristic peak or shoulder observed in the spectral region highlighted in pink in Fig. $4 \mathrm{~d}, \mathrm{~h}$ and 1 . This peak is at a lower wavenumber than the $v \mathrm{C}=\mathrm{O}$ peak of the surrounding PLA.

Resonant Mie scattering by particles of this size can contribute to such spectral peak shifts in the mid-IR (Bassan et al. 2010), however this can be ruled out for these data since the high wavenumber edge of the carbonyl absorbance peak around $1800 \mathrm{~cm}^{-1}$ (Fig. 3e) does not show 
any distortion usually associated with such scattering effects. We propose therefore that for ACNCC particles blended in PLA the absence of a peak at $1742 \mathrm{~cm}^{-1}$ corresponding to the unblended AC-NCC, and the presence instead of a strong carbonyl shoulder between $1739 \mathrm{~cm}^{-1}$ and $1754 \mathrm{~cm}^{-1}$ arise from (a) a loss of inter- and intramolecular hydrogen bonding in the ACNCC, and (b) an intermolecular interaction between the AC-NCC particles and PLA matrix through the carbonyl groups of these two components. Intramolecular carbonyl group interactions have previously been investigated for their role in the stabilization of proteins and peptides (Barlett et al. 2010), whereby the main chain carbonyl group engages in a $\mathrm{C}=\mathrm{O} \ldots . . \mathrm{C}=\mathrm{O}$ interaction with another main chain carbonyl group in common secondary structures (Barlett et al. 2010 and Kamer et al. 2012). An $n \rightarrow \pi^{\star}$ interaction has been proposed whereby a lone pair ( $n$ ) of electrons from one carbonyl is delocalized into the antibonding orbital $\left(\pi^{\star}\right)$ of a neighbouring carbonyl, resulting in structural stabilisation. Such $n \rightarrow \pi^{*}$ intramolecular interaction between carbonyl groups, as indicated in Fig. 5a, has also been proposed as a mechanism for stabilisation in PLA, where it was concluded that this type of interaction predominates in the absence of intramolecular hydrogen bonding (Newberry and Raines. 2013). It is therefore quite likely that when the acetyl groups are partially substituted on the surface of the NCC particles and these particles used as a filler in PLA, $n \rightarrow \pi^{\star}$ carbonyl interaction occurs between carbonyl groups present in PLA and those present in the acetylated NCC at the fibrematrix interface, as illustrated in Fig. 5b. These relatively weak $n \rightarrow \pi^{*}$ interactions can lead to a shift of the $\nu \mathrm{C}=\mathrm{O}$ peak position, as we have observed by second derivative analysis. The level of partial substitution achieved was not sufficient to prevent the aggregation of the AC-NCC into particles of a few microns in size, but the surface of these particles remain available for carbonyl interaction with the PLA within which they are embedded. Such carbonyl group interaction 
would result in a strong fiber-matrix interface region, thereby enhancing stress transfer mechanism between the acetylated cellulose and the PLA matrix.

Furthermore, the shoulder observed in PLA at around $1750 \mathrm{~cm}^{-1}$ (Fig. 3f, black) could possibly also arise from this interaction. It is known that by chemically manipulating the nature of the interfacial region, Gibbs free energy is minimized to reach the equilibrium state, thereby facilitating the interfacial adhesion between the fiber and the matrix (Flory. 1941). Furthermore, if steric repulsion caused by the acetyl groups attached to the NCC surface overcomes NCCNCC interaction, then modified NCC is more likely to be dispersed well in the hydrophobic matrix such as PLA. In other words, by manipulating the chemical nature of the interface, the fibre-matrix interfacial tension can be minimized to reach the equilibrium state, thereby improving the interfacial adhesion (Mukherjee et al. 2013 and Pei et al. 2010). It was also reported elsewhere that the increase in the density of ester linkages from the polymer matrix to the cellulose nanocrystal interface contributed to the average increase in elastic modulus of the interphase region in poly (vinyl alcohol) (PVA)-poly (acrylic acid) (PAA)-cellulose nanocrystal (CNC) (Pakzad et al. 2012). Thus determining the spatial distribution of the different components of the composites, and understanding their chemical interaction, can be crucial in understanding the behavior of network formation and the improvement in mechanical properties of the composite.

\section{Conclusions}

The spatial distribution of the different functional group chemistry of the acetylated nanocrystalline cellulose based PLA composite (10 wt. \%) has been revealed by FTIR chemical 
imaging at the Australian Synchrotron, and has proven effective in helping to understand the nature of the ester linkage between the substrate and functionalised filler. While incomplete acetylation enables some aggregation to occur between nanocrystals, a weak carbonyl group interaction at the interface region is shown to occur, as revealed by second derivative analysis of the FTIR absorbance spectra of individual micron-scale filler particles. This kind of interaction is a possible basis for an enhanced stress transfer mechanism between cellulose and the PLA matrix, as reported earlier of the interphase region in poly (vinyl alcohol) (PVA)-poly (acrylic acid) (PAA)-cellulose nanocrystal (CNC). This may explain the enhanced functional properties of the PLA - AC-NCC composite. 


\section{Acknowledgements}

The authors would like to acknowledge the Australian Synchrotron for supporting access to the IR Microspectroscopy beamline, at which part of the data presented were collected, the scientific and technical assistance of Prof Frances Separovic for providing us the facility to conduct solid state NMR at Bio21 Molecular Science and Biotechnology Institute, The University of Melbourne, Mr Phil Francis and Mr Peter Rummel, of the Australian Microscopy and Microanalysis Research Facility at the RMIT Microscopy and Microanalysis Facility, RMIT University, Mr Frank Antolosic and Mr Mike Allan, from the Department of Chemical Engineering and Chemistry, RMIT University, for their continued support in completing the experimental work through an Australian Post Graduate Award. 


\section{References}

Bartlett GL, Choudhary A, Raines RT, Woolfson DN (2010) $n \rightarrow \pi^{*}$ interactions in proteins. Nat. Chem Biology 6: 615-620.doi: 10.1038/nchembio.406

Bassan P, Kohler A, Martens H, Lee J, Byrne HJ, Dumas P, Ehsan G, Brown, M, Clarke N, Gardner P (2010) Resonant Mie scattering (RMieS) correction of infrared spectra from highly scattering biological samples. Analyst 135: 268-277.doi.: 10.1039/b921056c

Braun B, Dorgan JR (2009) Single-step method for the isolation and surface functionalization of cellulosic nanowhiskers. Biomacromolecules 10: 334-341. doi: $10.1021 / \mathrm{bm} 8011117$

Ching YC, Ali ME, Abdullah LC, Choo KW, Kuan YC Julaihi SJ, Chuah CH, Liou N-S (2016) Rheological properties of cellulose nanocrystal-embedded polymer composites: a review. Cellulose 23: 1011-1030. doi: 10.1007/s10570-016-0868-3

Ciprari D, Jacob K, Tannenbaum R (2006) Characterization of polymer nanocomposite interphase and its impact on mechanical properties. Macromolecules 39: 6565-6573. doi: $10.1021 / \mathrm{ma} 0602270$

Clemons C, Sedlmair J, Illman B, Ibach R, Hirschmugl C (2013) Chemically imaging the effects of the addition of nanofibrillated cellulose on the distribution of poly (lactic acid) in poly (vinyl alcohol). Polymer 54: 2058-2061.doi: 10.1016/j.polymer.2013.02.016

Duncan WD, Williams GP (1983) Infrared synchrotron radiation from electron storage rings. Applied Optics 22: 2914-2923. doi: 10.1364/AO.22.002914 
Eichorn S, Dufresne A, Aranguren M, Marcovich N, Capadona J, Rowan S, Weder C, et al. ( 2010) Review: current international research into cellulose nanofibres and nanocomposites. Journ. of Mat Sci 45:1-33. doi: 10.1007/s10853-009-3874-0

Evans GR, Niederbichler AD, Chauvin P, Herman S, Bogle M, Otta L, Wang B, Patrick Jr CW (2000) Clinical long term in vivo evaluation of poly (L-Lactic acid) porous conduits for peripheral nerve regeneration. Journal of Biomaterial Science Polymer Edition 11:869878. doi: $10.1163 / 156856200744066$

Flory PJ (1941) Thermodynamics of high polymer solutions. Journal of Chemical Physics 9:660.doi:10.106 3/1.1723621

Heraud P, Caine S, Campanale N, Karnezis T, McNaughton D, Wood BR, Tobin MJ, Bernard CCA (2010) Early detection of the chemical changes occurring during the induction and prevention of autoimmune-mediated demyelination detected by FT-IR imaging. NeuroImage 49: 1180-1189. doi: 10.1016/j.neuroimage.2009.09.053

Huda MS, Drzal LT, Misra M, Mohanty AK (2006) Wood-fiber-reinforced poly(lactic acid) composites: Evaluation of the physicomechanical and morphological properties. Jour of App. Polym. Sci 102: 4856-4869. doi:_10.1002/app.24829

Kamer KJ, Choudhary A, Raines RT (2012) Intimate Interactions with Carbonyl Groups: Dipole-Dipole or $n \rightarrow \pi^{*}$ ? Journal of Organic Chemistry 78: 2099-2103.doi: 10.1021/jo302265k 
Kashiwagi T, Du F, Douglas JF, Winey KI, Harris RH, Shields JR (2005) Nanoparticle networks reduce the flammability of polymer nanocomposites . Nat Mater 4:928-933. doi: $10.1038 /$ nmat 1502

Khare HS, Burris DL (2010) A quantitative method for measuring nanocomposite dispersion. Polymer 51:719-729. doi: 10.1016/j.polymer.2009.12.031

Koenig JL (1999) Spectroscopy of Polymers, $2^{\text {nd }}$ ed. Elsevier, Amsterdam, 1999.ISBN:0$444-10031-8$

Labardi M, Prevosto D, Nguyen KH, Capaccioli S, Lucchest M, Rolla P (2010) Local dielectric spectroscopy of nanocomposites materials interfaces. J Vac Sci Technol B 28:C4D11-C4D17. http://dx.doi.org/10.1116/1.3368597

Lin N, Huang J, Chang PR, Feng JY (2011) Surface acetylation of cellulose nanocrystals and its reinforcing function in poly (lactic acid). Carbohydrate Polymers 83: 1834-1842.doi: 10.1016/j.carbpol.2010.10.047

Lu JZ, Negulesau II, Wu Q (2005) Maleated wood-fiber/high density polyethylene composites: Coupling mechanisms and interfacial characterization, Composite Interphase. Composite Interphase 12: 125-140.doi: 10.1163/1568554053542133

Mukherjee T, Sani M, Kao N, Gupta RK, Quazi N, Bhattacharya S (2013) Improved dispersion of cellulose microcrystals in polylactic acid (PLA) based composites applying surface acetylation. Chemical Engineering 101: 655-662.doi: 10.1016/j.ces.2013.07.032 
Needleman A, Borders TL, Brinson LC, Flores VM, Schadler LS (2010) Effect of interphase region on debonding of a CNT reinforced polymer nanocomposites. Compos. Sci. Technol 70: 2207- 2215.doi: 10.1016/j.compscitech.2010.09.002

Newberry RW， Raines RT (2013) $n \rightarrow \pi^{*}$ interactions in poly(lactic acid) suggest a role in protein folding. Chemical Communications 49:7699-7701. doi: 10.1039/c3ec44317e.

Oksman K, Mathew AP, Bondeson D, Kvien I (2006) Manufacturing process of cellulose whiskers/polylactic acid nanocomposites, Composites Science and Technology. Compos Sci Technol 66: 2776-2784.doi: 10.1016/j.compscitech.2006.03.002

Osmond G, Boon JJ, Puskar L, Drennan J (2012) Metal Stearate Distributions in Modern Artists' Oil Paints: Surface and Cross Sectional Investigation of Reference Paint Films Using Conventional and Synchrotron Infrared Microspectroscopy. Appl. Spectrosc 66: 11361144.doi: $10.1366 / 12-06659$

Pakzad A, Simonsen J, Yassar RS (2012) Gradient of nanomechanical properties in the interphase of cellulose nanocrystal composites. Comp. Sci. Tech. 72: 314-319.doi: 10.1016/j.compscitech.2011.11.020

Pei A, Zhou Q, Berglund LA (2010) Functionalized cellulose nanocrystals as biobased nucleation agents in poly(L-Lactide) (Plla) - crystallization and mechanical property effects, Compos. Sci. Technol 70:815-821.doi: 10.1016/j.compscitech.2010.01.018

Pracella M, Haque MU, Alvarez V (2010) Functionalization, Compatibilization and Properties of Polyolefin Composites with Natural Fibers. Polymers 2: 554-574.doi: $10.3390 /$ polym 2040554 
Priestley RD, Ellison CJ, Broadbelt LJ, Torkelson JM (2005) Structural relaxation of polymer glasses at surfaces, interfaces and in between. Science 309:456-459. doi: 10.1126/science. 1112217

Rittingstein P, Priestly RD, Broadbelt LJ, Torkelson JM (2007) Model polymer nanocomposites provide an understanding of confinement effects in real nanocomposites. Nat Mater 6: 278-282. doi:10.1038/nmat1870

Rittingstein P, Torkelson JM (2006) Polymer-nanoparticle interfacial interactions in polymer nanocomposites: confinement effects on glass transition temperature and suppression of physical aging. J Polym Sci Part B: Polym. Phys 44: 2935-2943. doi: $: 10.1038 /$ nmat 1870

Roth CB, Jager KL, Torkelson JM (2007) Eliminating the enhanced mobility at the free surface of polystyrene: fluororescence studies of the glass transition temperature in thin bilayer films of immiscible polymers. Macromolecules 40: 2568-2574. doi: $10.1021 / \mathrm{ma} 062864 \mathrm{w}$

Roy M, Nelson JK, MacCrone RK, Schadler LS (2005) Nanocomposite dielectrics-the role of the interface. IEEE Trans. Dielectr. Electr. Insul 12: 629-643. doi:

10.1109/TDEI.2005.1511089

Russell RA, Darwish TA, Puskar L, Martin DE, Holden PE, Foster LJR (2014) Deuterated polymers for probing phase separation using Infrared microspectroscopy Biomacromolecules 15:644-649.doi: 10.1021/bm4017012 
Široký J. Blackburn RS, Bechtold T, Taylor J, White P (2010) Attenuated total reflectance Fourier-transform Infrared spectroscopy analysis of crystallinity changes in lyocell following continuous treatment with sodium hydroxide. Cellulose 17: 103-115. doi: 10.1007/s10570009-9378-x

Tobin MJ, Puskar L, Barber RL, Harvey EC, Heraud P, Wood BR, Bambery KR, Dillon CT, Munro KL (2010) FTIR spectroscopy of single live cells in aqueous media by synchrotron IR microscopy using microfabricated sample holders. Vibrational Spectroscopy 53: 34-38.doi: 10.1016/j.vibspec.2010.02.005

Wang Y, Yao X, Parthasarathy R (2010) Characterization of interfacial Chemistry of Adhesive/Dentin Bond Using FTIR Chemical Imaging With Univariate and Multivariate Data Processing. J Biomed Mater Res A 91: 251-262.doi:_10.1002/jbm.a.32249 


\section{Figure captions}

Fig. 1 Acetylation of nanocrystalline cellulose. (a) Reaction scheme of acetylation of cellulose. (b) FTIR Absorbance spectra of pure NCC (green) and AC-NCC (blue) recorded in a diamond Micro Compression cell using $\mathrm{KBr}$ reference. Arrows indicate absorption peaks in AC-NCC from $\mathrm{C}=\mathrm{O}$ at $1742 \mathrm{~cm}^{-1}$, COO- at $1573 \mathrm{~cm}^{-1}$ and $\mathrm{C}-\mathrm{O}-\mathrm{C}$ at $1237 \mathrm{~cm}^{-1}$. AC-NCC structure is shown above for reference. (c) ${ }^{13} \mathrm{C} \mathrm{CP}-\mathrm{MAS}$ NMR spectra of pure NCC and acetylated NCC

Fig. 2 (a) Brightfield visible light micrograph of two AC-NCC crystals in PLA. (b) $v \mathrm{CH}_{2}$ absorption map of the two particles (outlined), generated by integrated peak area of IR absorbance between $2938 \mathrm{~cm}^{-1}$ and $2860 \mathrm{~cm}^{-1}$. (c) IR absorbance spectra of PLA (black) and ACNCC (orange), each spectrum averaged from the four similarly coloured points marked in Fig. 2b

Fig. 3 (a) Visible light image of microtomed section of AC-NCC in PLA. Inset shows outlined area of sample viewed with crossed polarizers, highlighting AC-NCC aggregate particles in white. White arrows indicate AC-NCC particles visible in both the polarized and unpolarized views. Black arrow indicates $\mathrm{KBr}$ reference. (b) Selected pair of AC-NCC aggregate particles (yellow square). (c) Higher magnification view of selected particles (outlined). (d) FTIR absorbance map of the area shown in (c), in which the colour scale indicates the integrated area of the $v \mathrm{C}=\mathrm{O}$ peak between $1739 \mathrm{~cm}^{-1}$ and $1754 \mathrm{~cm}^{-1}$. (e) FTIR absorbance spectra of PLA (black) and embedded AC-NCC particle (orange) with inset showing expanded view of $v \mathrm{C}=\mathrm{O}$ peak. Spectra are average from orange (AC-NCC) and black (PLA) points indicated in (d). (f) Inverted second derivative spectra of $\mathrm{vC}=\mathrm{O}$ absorbance peaks for PLA (black), AC-NCC (orange) and un-embedded pure AC-NCC (blue)

Fig. 4 Infrared absorbance maps and second derivative absorbance spectra of aggregate particles of acetylated and unacetylated nanocrystalline celulose in PLA matrix. a, e, i, m, q: visible light images of particles; b, f, j, n, r: $v \mathrm{CH}_{2}$ absorption maps and c, g, k, o, s: integrated $v \mathrm{C}=\mathrm{O}$ second derivative absorption peak areas of the same particles. $d, h, 1, p, t$, inverted second derivative absorption spectra of $\mathrm{v}=\mathrm{O}$ stretching peak for locations indicated by orange and black markers 
in $v \mathrm{C}=\mathrm{O}$ images. Arrows indicate position of $v \mathrm{C}=\mathrm{O}$ shoulder observed as strong in most $\mathrm{AC}$ NCC particles and weak or absent in NCC particles. Scale bar $=10 \mu \mathrm{m}$

Fig. 5 (a) Structure of PLA with curved arrow indicating putative intramolecular $n \rightarrow \pi^{*}$ interaction within PLA. (b) Example of proposed $n \rightarrow \pi^{*}$ interaction between carbonyl groups of PLA and AC-NCC, as highlighted 
(a)
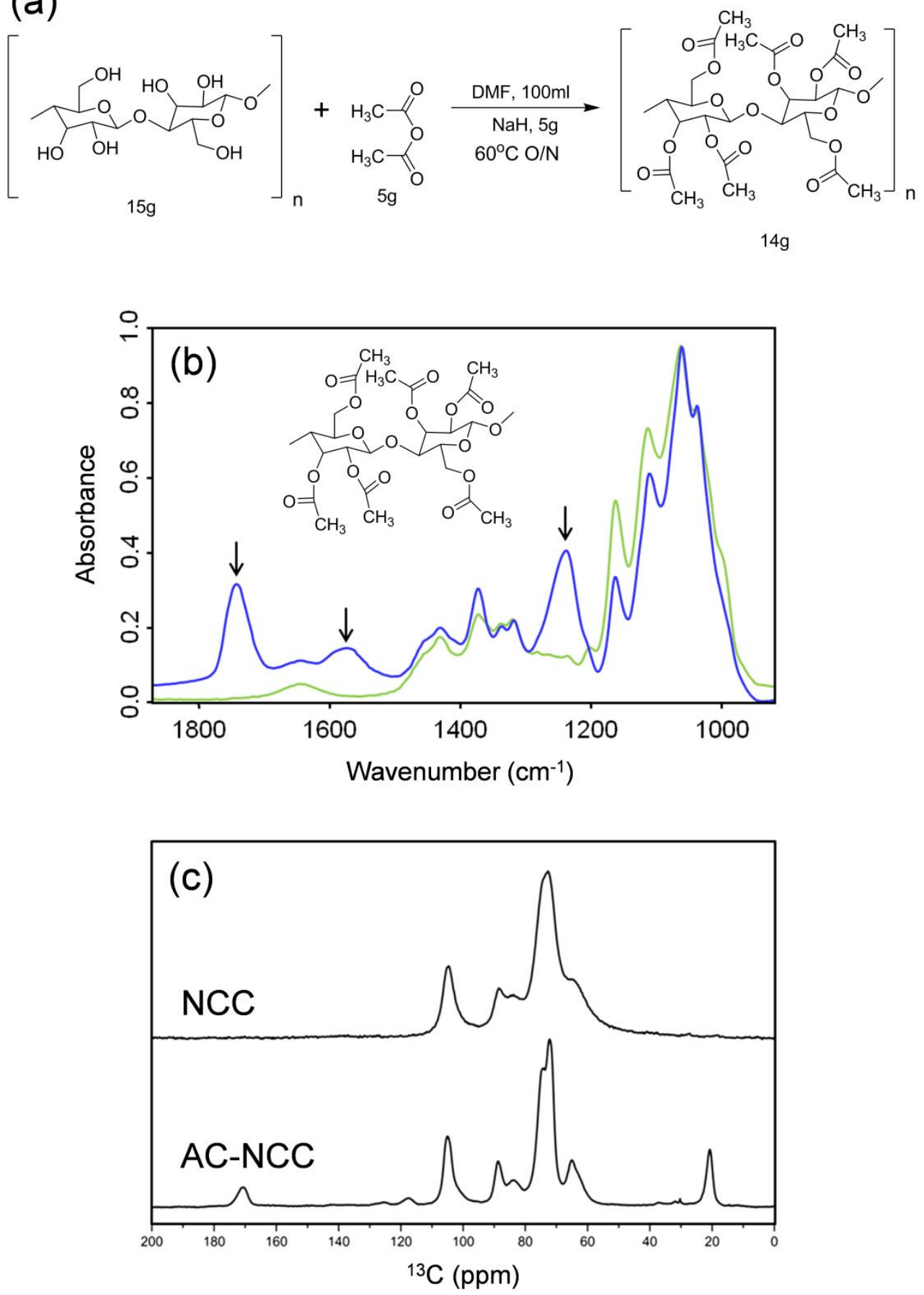

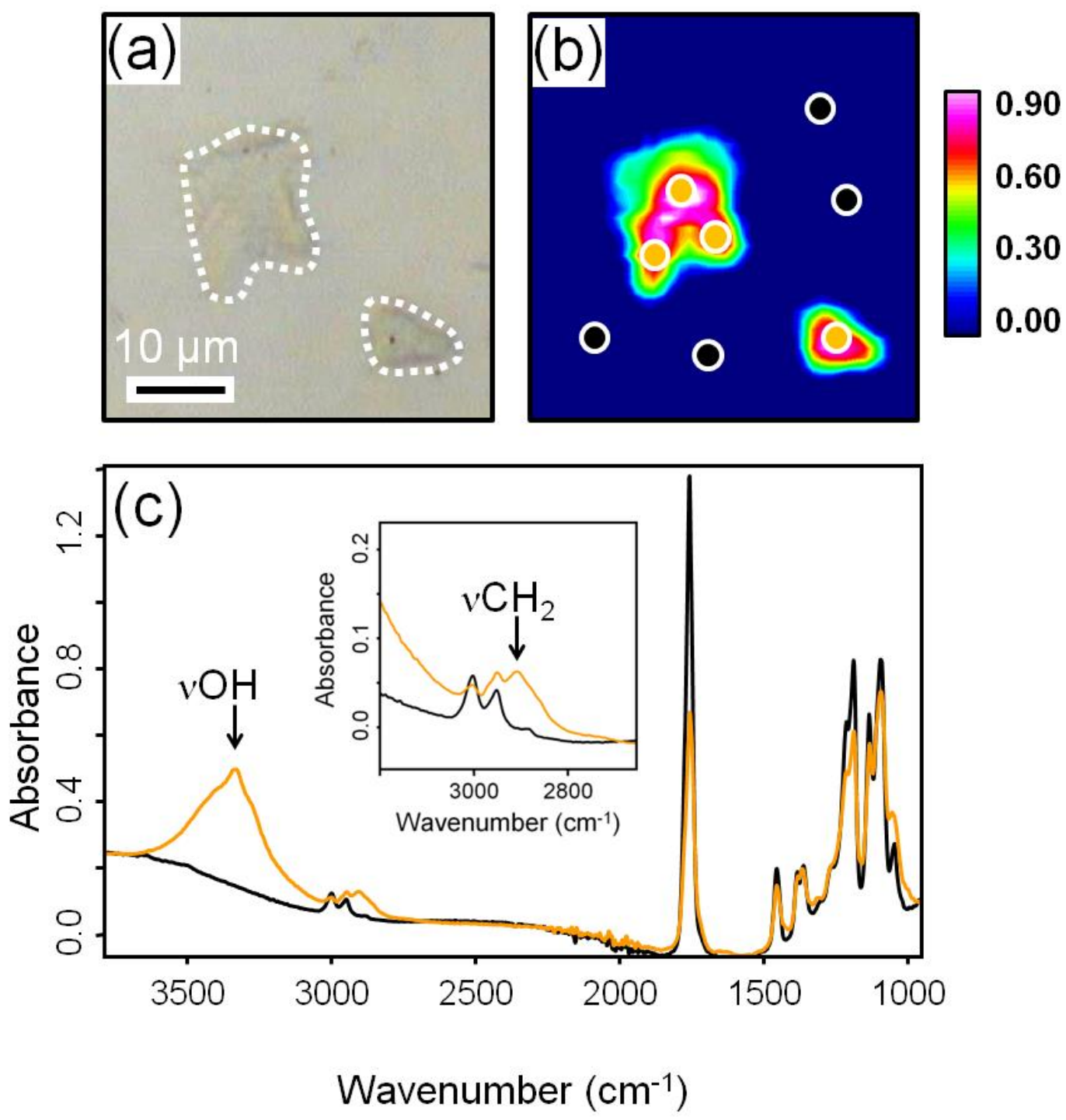

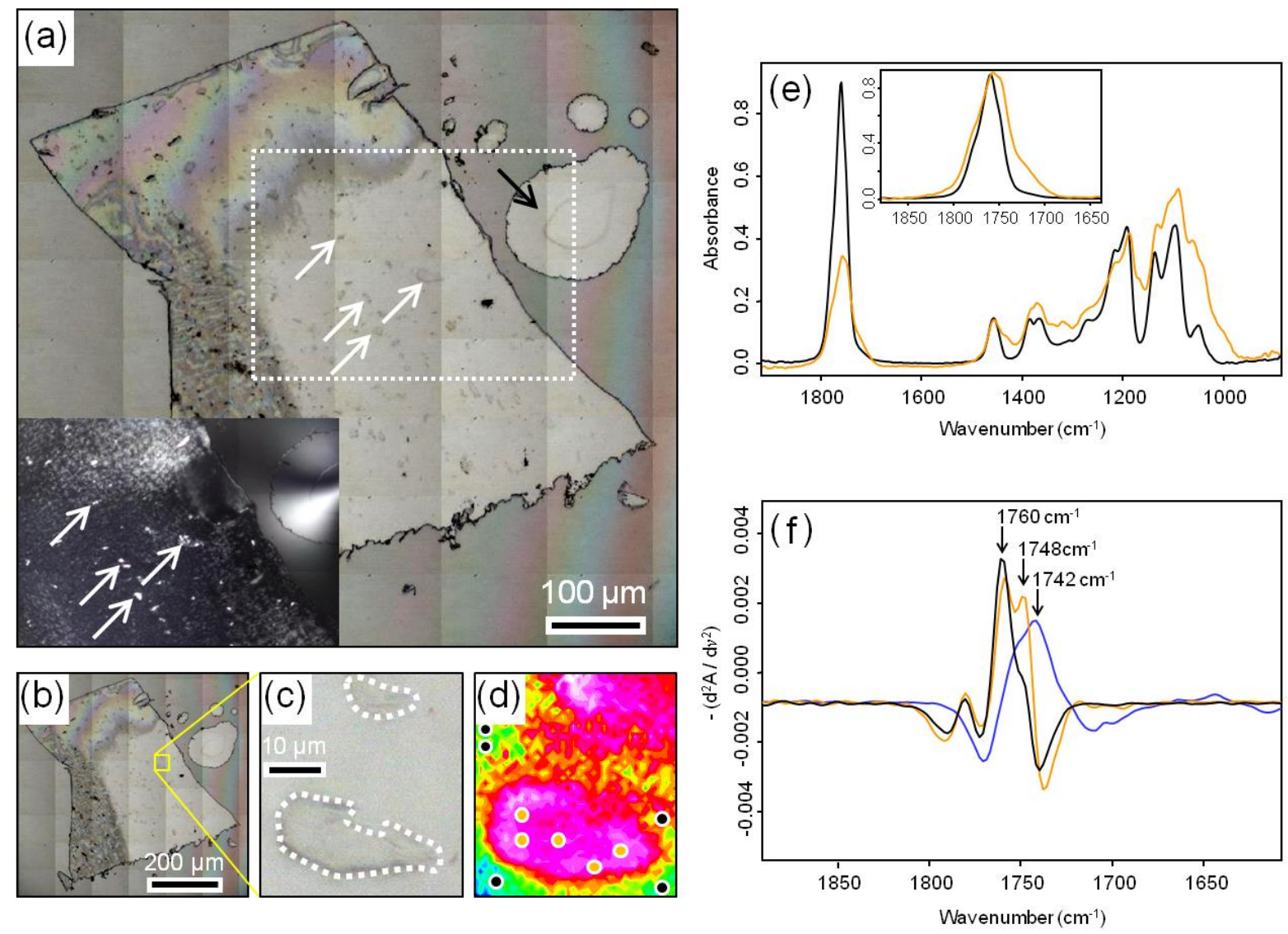

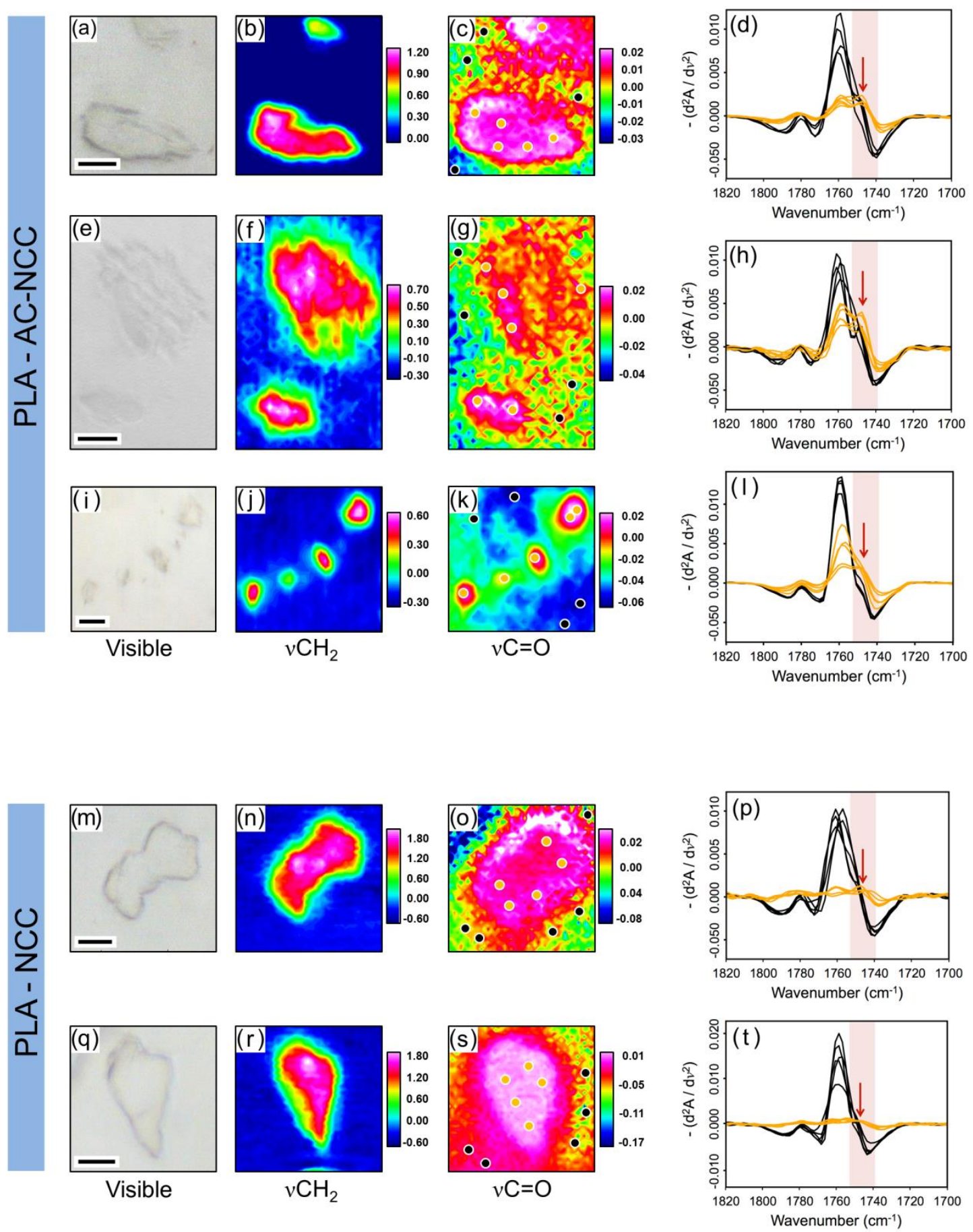


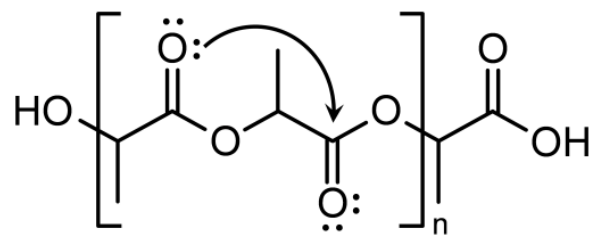

(a)

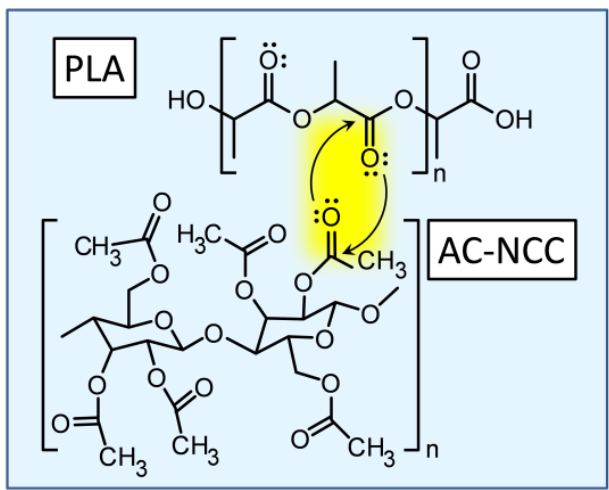

(b) 


\section{University Library}

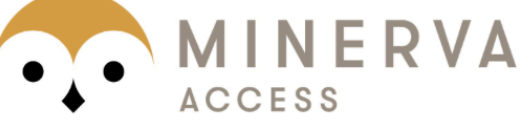

A gateway to Melbourne's research publications

Minerva Access is the Institutional Repository of The University of Melbourne

Author/s:

Mukherjee, T;Tobin, MJ;Puskar, L;Sani, M-A;Kao, N;Gupta, RK;Pannirselvam, M;Quazi,

$\mathrm{N}$;Bhattacharya, S

Title:

Chemically imaging the interaction of acetylated nanocrystalline cellulose (NCC) with a polylactic acid (PLA) polymer matrix

Date:

2017-04-01

Citation:

Mukherjee, T., Tobin, M. J., Puskar, L., Sani, M. -A., Kao, N., Gupta, R. K., Pannirselvam, M., Quazi, N. \& Bhattacharya, S. (2017). Chemically imaging the interaction of acetylated nanocrystalline cellulose (NCC) with a polylactic acid (PLA) polymer matrix. Cellulose, 24 (4), pp.1717-1729. https://doi.org/10.1007/s10570-017-1217-x.

Persistent Link:

http://hdl.handle.net/11343/283216 